Acta Crystallographica Section E

\section{Structure Reports}

Online

ISSN 1600-5368

\section{Diethylammonium 2,5-dihydroxy- benzenesulfonate}

\author{
William T. A. Harrison, ${ }^{a *}$ M. T. Swamy, ${ }^{b}$ H. S. Yathirajan, ${ }^{c}$ \\ M. A. Ashok ${ }^{c}$ and B. Narayana ${ }^{d}$
}

a Department of Chemistry, University of Aberdeen, Meston Walk, Aberdeen AB24 3UE, Scotland, 'bepartment of Chemistry, Sambhram Institute of Technology, Bangalore 560 097, India, 'Department of Studies in Chemistry, University of Mysore, Manasagangotri, Mysore 570 006, India, and ${ }^{\mathbf{d}}$ Department of Studies in Chemistry, Mangalore University, Mangalagangotri 574 199, India

Correspondence e-mail: w.harrison@abdn.ac.uk

Received 14 November 2007; accepted 18 November 2007

Key indicators: single-crystal X-ray study; $T=291 \mathrm{~K}$; mean $\sigma(\mathrm{C}-\mathrm{C})=0.003 \AA$; $R$ factor $=0.029 ; w R$ factor $=0.079 ;$ data-to-parameter ratio $=20.8$.

The title compound, $\mathrm{C}_{4} \mathrm{H}_{12} \mathrm{~N}^{+} \cdot \mathrm{C}_{6} \mathrm{H}_{5} \mathrm{O}_{5} \mathrm{~S}^{-}$, also known as ethamsylate, is a molecular salt. Anion-to-anion $\mathrm{O}-\mathrm{H} \cdots \mathrm{O}$ hydrogen bonds lead to helices containing $C(6)$ and $C(7)$ chains and further bonds of the same type cross-link the chains. Cation-to-anion $\mathrm{N}-\mathrm{H} \cdots \mathrm{O}$ hydrogen bonds complete the structure.

\section{Related literature}

For a related structure, see: Solans et al. (1982). For background, see: Harrison et al. (2007); Hernandez et al. (2004); Garay et al. (2006). For reference structural data, see: Allen et al. (1987). For hydrogen-bonding motifs, see: Bernstein et al. (1995).
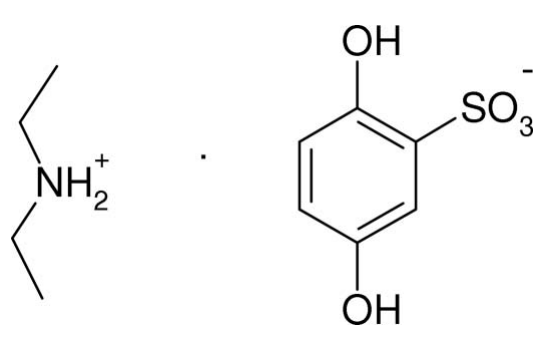

\section{Experimental}

\section{Crystal data}

$\mathrm{C}_{4} \mathrm{H}_{12} \mathrm{~N}^{+} \cdot \mathrm{C}_{6} \mathrm{H}_{5} \mathrm{O}_{5} \mathrm{~S}^{-}$

$M_{r}=263.31$

Trigonal, $P 3_{2}$

$a=8.7006$ (4) ^

$c=14.0732(6) \AA$

$V=922.62(7) \AA^{3}$

\section{Data collection \\ Bruker SMART1000 CCD \\ diffractometer \\ (SADABS; Bruker, 1999) \\ $T_{\min }=0.900, T_{\max }=0.926$ \\ Refinement \\ $R\left[F^{2}>2 \sigma\left(F^{2}\right)\right]=0.029$ \\ $w R\left(F^{2}\right)=0.079$ \\ $S=1.08$ \\ 3516 reflections \\ 169 parameters \\ 1 restraint}

Absorption correction: multi-scan
7627 measured reflections 3516 independent reflections 3307 reflections with $I>2 \sigma(I)$ $R_{\text {int }}=0.016$

$\mathrm{H}$ atoms treated by a mixture of independent and constrained refinement

$\Delta \rho_{\max }=0.22{\mathrm{e} \AA^{-3}}^{-3}$

Absolute structure: Flack (1983), with 1734 Friedel pairs Flack parameter: $0.03(5)$
$\Delta \rho_{\min }=-0.17{\mathrm{e} \AA^{-3}}^{-3}$

Table 1

Hydrogen-bond geometry $\left(\AA,^{\circ}\right)$.

\begin{tabular}{lllll}
\hline$D-\mathrm{H} \cdots A$ & $D-\mathrm{H}$ & $\mathrm{H} \cdots A$ & $D \cdots A$ & $D-\mathrm{H} \cdots A$ \\
\hline $\mathrm{O} 1-\mathrm{H} 1 \cdots \mathrm{O} 3^{\mathrm{i}}$ & $0.85(2)$ & $2.02(3)$ & $2.8441(17)$ & $163(2)$ \\
$\mathrm{O} 2-\mathrm{H} 2 \cdots \mathrm{O} 4^{\mathrm{ii}}$ & $0.75(3)$ & $1.90(3)$ & $2.6480(19)$ & $174(3)$ \\
$\mathrm{N} 1-\mathrm{H} 3 \cdots \mathrm{O} 3$ & $0.82(3)$ & $2.43(3)$ & $3.084(2)$ & $138(2)$ \\
$\mathrm{N} 1-\mathrm{H} 3 \cdots \mathrm{O}{ }^{\mathrm{iii}}$ & $0.82(3)$ & $2.49(3)$ & $3.0691(19)$ & $129(2)$ \\
$\mathrm{N} 1-\mathrm{H} 4 \cdots \mathrm{O} 5^{\text {iv }}$ & $0.96(3)$ & $1.94(3)$ & $2.888(2)$ & $171(2)$ \\
\hline
\end{tabular}

Symmetry codes: (i) $\quad-y+1, x-y, z-\frac{1}{3}$; $\quad$ (ii) $\quad-x+y+2,-x+1, z+\frac{1}{3}$; $\quad$ (iii) $-y+1, x-y-1, z-\frac{1}{3}$; (iv) $-y, x-y-1, z-\frac{1}{3}$.

Data collection: SMART (Bruker, 1999); cell refinement: SAINT (Bruker, 1999); data reduction: $S A I N T$; $\operatorname{program}(\mathrm{s})$ used to solve structure: SHELXS97 (Sheldrick, 1997); program(s) used to refine structure: SHELXL97 (Sheldrick, 1997); molecular graphics: ORTEP-3 (Farrugia, 1997); software used to prepare material for publication: SHELXL97.

MTS thanks Sambhram Institute of Technology for research facilities.

Supplementary data and figures for this paper are available from the IUCr electronic archives (Reference: FJ2077).

\section{References}

Allen, F. H., Kennard, O., Watson, D. G., Brammer, L., Orpen, A. G. \& Taylor, R. (1987). J. Chem. Soc. Perkin Trans. 2, pp. S1-19.

Bernstein, J., Davis, R. E., Shimoni, L. \& Chang, N.-L. (1995). Angew. Chem. Int. Ed. Engl. 34, 1555-1573.

Bruker (1999). SMART, SAINT and SADABS. Bruker AXS Inc., Madison, Wisconsin, USA.

Farrugia, L. J. (1997). J. Appl. Cryst. 30, 565.

Flack, H. D. (1983). Acta Cryst. A39, 876-881.

Garay, R. P., Chiavaroli, C. \& Hannaert, P. (2006). Am. J. Ther. 13, 236-247.

Harrison, W. T. A., Yathirajan, H. S., Bindya, S., Anilkumar, H. G. \& Devaraju (2007). Acta Cryst. C63, o129-o131.

Hernandez, M. R., Alvarez-Guerra, M., Escolar, G., Chiavaroli, C., Hannaert, P. \& Garay, R. P. (2004). Fundam. Clin. Pharmacol. 18, 423-430.

Sheldrick, G. M. (1997). SHELXS97 and SHELXL97. University of Göttingen, Germany.

Solans, X., Plana, F. \& Font-Altaba, M. (1982). Acta Cryst. B38, 651-653.

Spek, A. L. (2003). J. Appl. Cryst. 36, 7-13. 


\section{supplementary materials}


Acta Cryst. (2007). E63, o4870 [ doi:10.1107/S1600536807060540 ]

\title{
Diethylammonium 2,5-dihydroxybenzenesulfonate
}

\author{
W. T. A. Harrison, M. T. Swamy, H. S. Yathirajan, M. A. Ashok and B. Narayana
}

\section{Comment}

The title compound, (I), ethamsylate, or diethylammonium 2,5-dihydroxybenzenesulfonate, $\mathrm{C}_{4} \mathrm{H}_{12} \mathrm{~N}^{+} \cdot \mathrm{C}_{6} \mathrm{H}_{5} \mathrm{O}_{5} \mathrm{~S}^{-}$, is a drug known to possess antihemorrhagic properties. It is said to correct abnormal platelet adhesion, thereby reducing capillary bleeding (Hernandez et al., 2004; Garay et al., 2006).

In continuation of our work on crystal structures of pharmaceutical compounds (Harrison et al., 2007) and in view of the importance of (I), its crystal structure is now reported. The crystal structure of the related bis(diethylammonium) 2,5-dihydroxy-1,4-benzenedisulfonate, $\left(\mathrm{C}_{4} \mathrm{H}_{12} \mathrm{~N}^{+}\right)_{2} \cdot \mathrm{C}_{6} \mathrm{H}_{4} \mathrm{O}_{2} \mathrm{~S}_{2}{ }^{2-}$, was reported earlier (Solans et al., 1982).

Compound (I) formally arises via proton transfer from the sulfonic acid group to the secondary amine $\mathrm{N}$ atom (Fig. 1) to yield a molecular salt. There are no previous crystal structures containg the 2,5-dihydroxybenzenesulfonate anion, but its individual bond lengths and angles are similar to the corresponding values in the related 2,5-dihydroxy-1,4-benzenedisulfonate (Solans et al., 1982). Overall, the geomerical parameters of the two components in (I) may be regarded as normal (Allen et al., 1987).

Compound (I) crystallizes in the chiral space group P32, with a well defined absolute structure (Flack, 1983). The constituents are achiral, so the enantiomorphic nature of the structure must arise from the packing, and an equal number of enantiomers (crystallizing in $\mathrm{P}_{1}$ and $\mathrm{P} 3_{2}$ ) must exist in the bulk sample. The packing is influenced by $\mathrm{O}-\mathrm{H} \cdots \mathrm{O}$ and $\mathrm{N}-\mathrm{H} \cdots \mathrm{O}$ hydrogen bonds (Table 1). Considered in isolation, both the $\mathrm{O} 1-\mathrm{H} 1 \cdots \mathrm{O} 3^{\mathrm{i}}$ and $\mathrm{O} 2-\mathrm{H} 2 \cdots \mathrm{O} 4^{\mathrm{ii}}$ bonds (see Table 1 for symmetry codes) lead to helical chains propagating in [001]. The first of these results in a $\mathrm{C}(6)$ chain, the second in a $\mathrm{C}(7)$ chain (Fig. 2). Together, the $\mathrm{O}-\mathrm{H} \cdots \mathrm{O}$ bonds lead to a three-dimensional array. A strong, near linear $\mathrm{N}-\mathrm{H} \cdots \mathrm{O}$ and a weak, bifurcated $\mathrm{N}-\mathrm{H} \cdots(\mathrm{O}, O)$ hydrogen bond, which link the cation to adjacent anions, complete the structure.

\section{Experimental}

The title compound was obtained as a gift sample from Arvee Chem Pharma, Mysore, India. Crystals of (I) were grown by slow evaporation of an ethanol solution (m.p.: $400 \mathrm{~K}$ ).

\section{Refinement}

The $\mathrm{N}$ - and O-bound $\mathrm{H}$ atoms were located in a difference map and their positions were freely refined with $U_{\text {iso }}(\mathrm{H})=$ $1.2 U_{\text {eq }}$ (carrier). The C-bound $\mathrm{H}$ atoms were geometrically placed $(\mathrm{C}-\mathrm{H}=0.93-0.98 \AA)$ and refined as riding with $U_{\text {iso }}(\mathrm{H})=$ $1.2 U_{\text {eq }}$ (carrier) or $1.5 U_{\text {eq }}$ (methyl C). The methyl groups were allowed to rotate, but not to tip, to best fit the electron density. 


\section{supplementary materials}

\section{Figures}

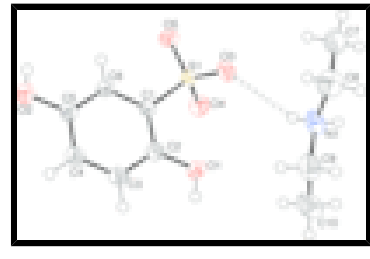

Fig. 1. View of the molecular structure of (I) showing 50\% displacement ellipsoids (arbitrary sphere for the $\mathrm{H}$ atom). The hydrogen bond is shown as a double-dashed line.

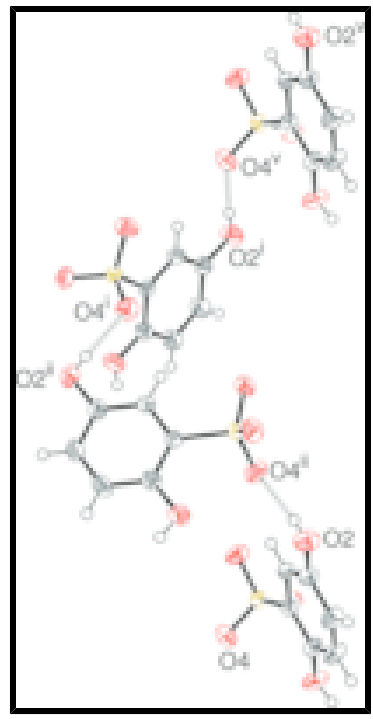

Fig. 2. Part of a helical chain on anions in the crystal structure of (I). The hydrogen bonds are shown as a double-dashed lines. Symmetry codes as in Table 1; additionally: (v) $x, y, z+1$.

\section{Diethylammonium 2,5-dihydroxybenzenesulfonate}

\section{Crystal data}

$\mathrm{C}_{4} \mathrm{H}_{12} \mathrm{~N}^{+} \cdot \mathrm{C}_{6} \mathrm{H}_{5} \mathrm{O}_{5} \mathrm{~S}^{-}$

$M_{r}=263.31$

Trigonal, $P 3_{2}$

Hall symbol: P 32

$a=8.7006(4) \AA$

$b=8.7006$ (4) $\AA$

$c=14.0732(6) \AA$

$\alpha=90^{\circ}$

$\beta=90^{\circ}$

$\gamma=120^{\circ}$

$V=922.62(7) \AA^{3}$

\section{Data collection}

Bruker SMART1000 CCD

diffractometer

Radiation source: fine-focus sealed tube

Monochromator: graphite
$Z=3$

$F_{000}=420$

$D_{\mathrm{x}}=1.422 \mathrm{Mg} \mathrm{m}^{-3}$

Mo $K \alpha$ radiation

$\lambda=0.71073 \AA$

Cell parameters from 5152 reflections

$\theta=2.7-29.9^{\circ}$

$\mu=0.27 \mathrm{~mm}^{-1}$

$T=291$ (2) K

Chunk, colourless

$0.40 \times 0.35 \times 0.30 \mathrm{~mm}$

3516 independent reflections

3307 reflections with $I>2 \sigma(I)$

$R_{\text {int }}=0.016$ 


\section{$T=291(2) \mathrm{K}$}

$\omega$ scans

Absorption correction: multi-scan

(SADABS; Bruker, 1999)

$T_{\min }=0.900, T_{\max }=0.926$

7627 measured reflections

$$
\begin{aligned}
& \theta_{\max }=30.0^{\circ} \\
& \theta_{\min }=2.7^{\circ} \\
& h=-12 \rightarrow 8 \\
& k=-10 \rightarrow 12 \\
& l=-19 \rightarrow 19
\end{aligned}
$$

\section{Refinement}

Refinement on $F^{2}$

Least-squares matrix: full

$R\left[F^{2}>2 \sigma\left(F^{2}\right)\right]=0.029$

$w R\left(F^{2}\right)=0.079$

$S=1.08$

3516 reflections

169 parameters

1 restraint

Primary atom site location: structure-invariant direct methods
Hydrogen site location: difmap and geom

$\mathrm{H}$ atoms treated by a mixture of independent and constrained refinement

$$
w=1 /\left[\sigma^{2}\left(F_{\mathrm{o}}{ }^{2}\right)+(0.0492 P)^{2}+0.0286 P\right]
$$

where $P=\left(F_{\mathrm{o}}^{2}+2 F_{\mathrm{c}}{ }^{2}\right) / 3$

$(\Delta / \sigma)_{\max }<0.001$

$\Delta \rho_{\max }=0.22$ e $\AA^{-3}$

$\Delta \rho_{\min }=-0.16 \mathrm{e} \AA^{-3}$

Extinction correction: SHELXL97 (Sheldrick, 1997),

$\mathrm{Fc}^{*}=\mathrm{kFc}\left[1+0.001 \mathrm{xFc}^{2} \lambda^{3} / \sin (2 \theta)\right]^{-1 / 4}$

Extinction coefficient: 0.028 (3)

Absolute structure: Flack (1983), with 1734 Friedel

pairs

Secondary atom site location: difference Fourier map Flack parameter: 0.03 (5)

\section{Special details}

Geometry. All e.s.d.'s (except the e.s.d. in the dihedral angle between two 1.s. planes) are estimated using the full covariance matrix. The cell e.s.d.'s are taken into account individually in the estimation of e.s.d.'s in distances, angles and torsion angles; correlations between e.s.d.'s in cell parameters are only used when they are defined by crystal symmetry. An approximate (isotropic) treatment of cell e.s.d.'s is used for estimating e.s.d.'s involving l.s. planes.

Refinement. Refinement of $\mathrm{F}^{2}$ against ALL reflections. The weighted $R$-factor $w R$ and goodness of fit $\mathrm{S}$ are based on $\mathrm{F}^{2}$, conventional $R$-factors $R$ are based on $\mathrm{F}$, with $\mathrm{F}$ set to zero for negative $\mathrm{F}^{2}$. The threshold expression of $\mathrm{F}^{2}>2$ sigma( $\left(\mathrm{F}^{2}\right)$ is used only for calculating $R$-factors(gt) etc. and is not relevant to the choice of reflections for refinement. $R$-factors based on $\mathrm{F}^{2}$ are statistically about twice as large as those based on F, and R- factors based on ALL data will be even larger.

Fractional atomic coordinates and isotropic or equivalent isotropic displacement parameters $\left(\AA^{2}\right)$

$\begin{array}{lllll} & x & y & z & U_{\text {iso }} * / U_{\text {eq }} \\ \text { C1 } & 0.92539(18) & 0.24787(18) & 0.04160(9) & 0.0255(2) \\ \text { C2 } & 0.96186(19) & 0.3460(2) & -0.04236(11) & 0.0303(3) \\ \text { C3 } & 1.1272(2) & 0.4984(2) & -0.05275(13) & 0.0379(3) \\ \text { H3A } & 1.1536 & 0.5643 & -0.1084 & 0.046^{*} \\ \text { C4 } & 1.2525(2) & 0.5526(2) & 0.01894(12) & 0.0379(4) \\ \text { H4A } & 1.3625 & 0.6544 & 0.0110 & 0.045^{*} \\ \text { C5 } & 1.2151(2) & 0.45559(19) & 0.10314(11) & 0.0304(3) \\ \text { C6 } & 1.05126(19) & 0.30364(19) & 0.11403(10) & 0.0268(3) \\ \text { H6 } & 1.0251 & 0.2385 & 0.1700 & 0.032 *\end{array}$


supplementary materials

\begin{tabular}{|c|c|c|c|c|}
\hline $\mathrm{O} 1$ & $0.83456(17)$ & 0.28992 (19) & $-0.11154(9)$ & $0.0413(3)$ \\
\hline H1 & $0.879(3)$ & $0.367(3)$ & $-0.1556(17)$ & $0.050^{*}$ \\
\hline $\mathrm{O} 2$ & $1.34355(16)$ & $0.51443(18)$ & $0.17195(9)$ & $0.0413(3)$ \\
\hline $\mathrm{H} 2$ & $1.305(4)$ & $0.454(4)$ & $0.2142(19)$ & $0.050 *$ \\
\hline $\mathrm{S} 1$ & $0.72428(4)$ & 0.04463 (4) & $0.05746(2)$ & $0.02561(9)$ \\
\hline $\mathrm{O} 3$ & $0.57761(15)$ & $0.08132(17)$ & $0.05299(8)$ & $0.0369(3)$ \\
\hline $\mathrm{O} 4$ & $0.71698(18)$ & $-0.07059(17)$ & $-0.02011(9)$ & $0.0399(3)$ \\
\hline $\mathrm{O} 5$ & $0.73716(16)$ & $-0.02270(16)$ & $0.14986(8)$ & $0.0383(3)$ \\
\hline $\mathrm{C} 7$ & $0.1436(3)$ & $-0.0537(3)$ & $0.03267(17)$ & $0.0538(5)$ \\
\hline H7A & 0.0917 & -0.0234 & 0.0844 & $0.081 *$ \\
\hline H7B & 0.2264 & -0.0862 & 0.0572 & $0.081^{*}$ \\
\hline $\mathrm{H} 7 \mathrm{C}$ & 0.0519 & -0.1517 & -0.0020 & $0.081 *$ \\
\hline $\mathrm{C} 8$ & 0.2377 (3) & 0.1017 (3) & $-0.03175(17)$ & $0.0551(5)$ \\
\hline $\mathrm{H} 8 \mathrm{~A}$ & 0.1524 & 0.1297 & -0.0592 & $0.066^{*}$ \\
\hline H8B & 0.3221 & 0.2037 & 0.0049 & $0.066^{*}$ \\
\hline C9 & $0.4290(3)$ & $0.2214(3)$ & $-0.17701(17)$ & $0.0513(5)$ \\
\hline H9A & 0.3551 & 0.2732 & -0.1905 & $0.062^{*}$ \\
\hline H9B & 0.5373 & 0.3120 & -0.1473 & $0.062 *$ \\
\hline $\mathrm{C} 10$ & $0.4738(3)$ & $0.1650(3)$ & $-0.2678(2)$ & $0.0615(6)$ \\
\hline H10A & 0.5233 & 0.2628 & -0.3115 & $0.092 *$ \\
\hline H10B & 0.3682 & 0.0682 & -0.2946 & $0.092 *$ \\
\hline $\mathrm{H} 10 \mathrm{C}$ & 0.5588 & 0.1276 & -0.2557 & $0.092 *$ \\
\hline N1 & $0.3328(2)$ & $0.0685(2)$ & $-0.10904(12)$ & $0.0427(3)$ \\
\hline H3 & $0.401(4)$ & $0.038(4)$ & $-0.0891(18)$ & $0.051 *$ \\
\hline H4 & $0.238(4)$ & $-0.038(4)$ & $-0.1357(17)$ & $0.051^{*}$ \\
\hline
\end{tabular}

Atomic displacement parameters $\left(\AA^{2}\right)$

$\begin{array}{lllllll} & U^{11} & U^{22} & U^{33} & U^{12} & U^{13} & U^{23} \\ \text { C1 } & 0.0235(6) & 0.0256(6) & 0.0259(6) & 0.0113(5) & 0.0015(5) & 0.0008(5) \\ \text { C2 } & 0.0293(7) & 0.0332(7) & 0.0295(6) & 0.0165(6) & 0.0000(5) & 0.0052(6) \\ \text { C3 } & 0.0343(7) & 0.0359(8) & 0.0384(8) & 0.0136(6) & 0.0028(6) & 0.0152(6) \\ \text { C4 } & 0.0309(7) & 0.0303(7) & 0.0419(8) & 0.0073(6) & -0.0002(6) & 0.0094(6) \\ \text { C5 } & 0.0274(7) & 0.0290(6) & 0.0312(6) & 0.0113(6) & -0.0017(5) & 0.0003(6) \\ \text { C6 } & 0.0276(6) & 0.0274(6) & 0.0239(6) & 0.0127(5) & -0.0003(5) & 0.0012(5) \\ \text { O1 } & 0.0362(6) & 0.0485(7) & 0.0302(5) & 0.0144(5) & -0.0053(4) & 0.0098(5) \\ \text { O2 } & 0.0308(6) & 0.0401(6) & 0.0370(6) & 0.0058(5) & -0.0081(5) & -0.0009(5) \\ \text { S1 } & 0.02316(15) & 0.02566(15) & 0.02480(14) & 0.00979(12) & 0.00046(11) & -0.00058(12) \\ \text { O3 } & 0.0266(5) & 0.0431(6) & 0.0429(6) & 0.0190(5) & 0.0036(4) & 0.0021(5) \\ \text { O4 } & 0.0428(6) & 0.0370(6) & 0.0393(6) & 0.0195(5) & -0.0030(5) & -0.0125(5) \\ \text { O5 } & 0.0373(6) & 0.0349(6) & 0.0320(5) & 0.0100(5) & -0.0004(4) & 0.0096(4) \\ \text { C7 } & 0.0437(10) & 0.0623(13) & 0.0535(11) & 0.0251(9) & -0.0015(9) & -0.0083(9) \\ \text { C8 } & 0.0630(13) & 0.0535(11) & 0.0598(12) & 0.0373(11) & -0.0016(10) & -0.0107(9) \\ \text { C9 } & 0.0430(10) & 0.0388(9) & 0.0690(13) & 0.0180(8) & -0.0050(9) & 0.0055(9) \\ \text { C10 } & 0.0509(12) & 0.0484(11) & 0.0776(15) & 0.0190(10) & 0.0137(11) & 0.0141(11) \\ \text { N1 } & 0.0336(7) & 0.0376(7) & 0.0604(10) & 0.0204(6) & 0.0008(7) & -0.0020(7)\end{array}$


Geometric parameters $(\AA, \circ)$

\begin{tabular}{|c|c|c|c|}
\hline $\mathrm{C} 1-\mathrm{C} 6$ & 1.3937 (19) & $\mathrm{C} 7-\mathrm{C} 8$ & $1.488(4)$ \\
\hline $\mathrm{C} 1-\mathrm{C} 2$ & $1.3982(19)$ & $\mathrm{C} 7-\mathrm{H} 7 \mathrm{~A}$ & 0.9600 \\
\hline $\mathrm{C} 1-\mathrm{S} 1$ & $1.7732(14)$ & $\mathrm{C} 7-\mathrm{H} 7 \mathrm{~B}$ & 0.9600 \\
\hline $\mathrm{C} 2-\mathrm{O} 1$ & $1.3682(18)$ & $\mathrm{C} 7-\mathrm{H} 7 \mathrm{C}$ & 0.9600 \\
\hline $\mathrm{C} 2-\mathrm{C} 3$ & $1.393(2)$ & $\mathrm{C} 8-\mathrm{N} 1$ & $1.480(3)$ \\
\hline $\mathrm{C} 3-\mathrm{C} 4$ & $1.384(2)$ & $\mathrm{C} 8-\mathrm{H} 8 \mathrm{~A}$ & 0.9700 \\
\hline $\mathrm{C} 3-\mathrm{H} 3 \mathrm{~A}$ & 0.9300 & $\mathrm{C} 8-\mathrm{H} 8 \mathrm{~B}$ & 0.9700 \\
\hline $\mathrm{C} 4-\mathrm{C} 5$ & $1.396(2)$ & $\mathrm{C} 9-\mathrm{C} 10$ & $1.489(4)$ \\
\hline $\mathrm{C} 4-\mathrm{H} 4 \mathrm{~A}$ & 0.9300 & $\mathrm{C} 9-\mathrm{N} 1$ & $1.507(3)$ \\
\hline $\mathrm{C} 5-\mathrm{O} 2$ & $1.370(2)$ & C9-H9A & 0.9700 \\
\hline $\mathrm{C} 5-\mathrm{C} 6$ & $1.385(2)$ & C9-H9B & 0.9700 \\
\hline $\mathrm{C} 6-\mathrm{H} 6$ & 0.9300 & $\mathrm{C} 10-\mathrm{H} 10 \mathrm{~A}$ & 0.9600 \\
\hline $\mathrm{O} 1-\mathrm{H} 1$ & $0.85(2)$ & $\mathrm{C} 10-\mathrm{H} 10 \mathrm{~B}$ & 0.9600 \\
\hline $\mathrm{O} 2-\mathrm{H} 2$ & $0.75(3)$ & $\mathrm{C} 10-\mathrm{H} 10 \mathrm{C}$ & 0.9600 \\
\hline $\mathrm{S} 1-\mathrm{O} 5$ & $1.4533(12)$ & $\mathrm{N} 1-\mathrm{H} 3$ & $0.82(3)$ \\
\hline $\mathrm{S} 1-\mathrm{O} 4$ & $1.4618(12)$ & $\mathrm{N} 1-\mathrm{H} 4$ & $0.96(3)$ \\
\hline $\mathrm{S} 1-\mathrm{O} 3$ & $1.4635(13)$ & & \\
\hline $\mathrm{C} 6-\mathrm{C} 1-\mathrm{C} 2$ & $120.44(13)$ & $\mathrm{H} 7 \mathrm{~A}-\mathrm{C} 7-\mathrm{H} 7 \mathrm{~B}$ & 109.5 \\
\hline $\mathrm{C} 6-\mathrm{C} 1-\mathrm{S} 1$ & $117.84(10)$ & $\mathrm{C} 8-\mathrm{C} 7-\mathrm{H} 7 \mathrm{C}$ & 109.5 \\
\hline $\mathrm{C} 2-\mathrm{C} 1-\mathrm{S} 1$ & $121.65(11)$ & $\mathrm{H} 7 \mathrm{~A}-\mathrm{C} 7-\mathrm{H} 7 \mathrm{C}$ & 109.5 \\
\hline $\mathrm{O} 1-\mathrm{C} 2-\mathrm{C} 3$ & $122.09(14)$ & $\mathrm{H} 7 \mathrm{~B}-\mathrm{C} 7-\mathrm{H} 7 \mathrm{C}$ & 109.5 \\
\hline $\mathrm{O} 1-\mathrm{C} 2-\mathrm{C} 1$ & $119.18(13)$ & $\mathrm{N} 1-\mathrm{C} 8-\mathrm{C} 7$ & $112.22(18)$ \\
\hline $\mathrm{C} 3-\mathrm{C} 2-\mathrm{C} 1$ & $118.74(13)$ & $\mathrm{N} 1-\mathrm{C} 8-\mathrm{H} 8 \mathrm{~A}$ & 109.2 \\
\hline $\mathrm{C} 4-\mathrm{C} 3-\mathrm{C} 2$ & $120.70(14)$ & $\mathrm{C} 7-\mathrm{C} 8-\mathrm{H} 8 \mathrm{~A}$ & 109.2 \\
\hline $\mathrm{C} 4-\mathrm{C} 3-\mathrm{H} 3 \mathrm{~A}$ & 119.6 & $\mathrm{~N} 1-\mathrm{C} 8-\mathrm{H} 8 \mathrm{~B}$ & 109.2 \\
\hline $\mathrm{C} 2-\mathrm{C} 3-\mathrm{H} 3 \mathrm{~A}$ & 119.6 & $\mathrm{C} 7-\mathrm{C} 8-\mathrm{H} 8 \mathrm{~B}$ & 109.2 \\
\hline $\mathrm{C} 3-\mathrm{C} 4-\mathrm{C} 5$ & $120.46(14)$ & $\mathrm{H} 8 \mathrm{~A}-\mathrm{C} 8-\mathrm{H} 8 \mathrm{~B}$ & 107.9 \\
\hline $\mathrm{C} 3-\mathrm{C} 4-\mathrm{H} 4 \mathrm{~A}$ & 119.8 & $\mathrm{C} 10-\mathrm{C} 9-\mathrm{N} 1$ & $112.02(17)$ \\
\hline $\mathrm{C} 5-\mathrm{C} 4-\mathrm{H} 4 \mathrm{~A}$ & 119.8 & $\mathrm{C} 10-\mathrm{C} 9-\mathrm{H} 9 \mathrm{~A}$ & 109.2 \\
\hline $\mathrm{O} 2-\mathrm{C} 5-\mathrm{C} 6$ & $122.44(14)$ & $\mathrm{N} 1-\mathrm{C} 9-\mathrm{H} 9 \mathrm{~A}$ & 109.2 \\
\hline $\mathrm{O} 2-\mathrm{C} 5-\mathrm{C} 4$ & $118.29(13)$ & $\mathrm{C} 10-\mathrm{C} 9-\mathrm{H} 9 \mathrm{~B}$ & 109.2 \\
\hline $\mathrm{C} 6-\mathrm{C} 5-\mathrm{C} 4$ & $119.27(14)$ & $\mathrm{N} 1-\mathrm{C} 9-\mathrm{H} 9 \mathrm{~B}$ & 109.2 \\
\hline $\mathrm{C} 5-\mathrm{C} 6-\mathrm{C} 1$ & $120.39(13)$ & $\mathrm{H} 9 \mathrm{~A}-\mathrm{C} 9-\mathrm{H} 9 \mathrm{~B}$ & 107.9 \\
\hline $\mathrm{C} 5-\mathrm{C} 6-\mathrm{H} 6$ & 119.8 & $\mathrm{C} 9-\mathrm{C} 10-\mathrm{H} 10 \mathrm{~A}$ & 109.5 \\
\hline $\mathrm{C} 1-\mathrm{C} 6-\mathrm{H} 6$ & 119.8 & $\mathrm{C} 9-\mathrm{C} 10-\mathrm{H} 10 \mathrm{~B}$ & 109.5 \\
\hline $\mathrm{C} 2-\mathrm{O} 1-\mathrm{H} 1$ & $105.5(17)$ & $\mathrm{H} 10 \mathrm{~A}-\mathrm{C} 10-\mathrm{H} 10 \mathrm{~B}$ & 109.5 \\
\hline $\mathrm{C} 5-\mathrm{O} 2-\mathrm{H} 2$ & $108(2)$ & $\mathrm{C} 9-\mathrm{C} 10-\mathrm{H} 10 \mathrm{C}$ & 109.5 \\
\hline $\mathrm{O} 5-\mathrm{S} 1-\mathrm{O} 4$ & $112.17(8)$ & $\mathrm{H} 10 \mathrm{~A}-\mathrm{C} 10-\mathrm{H} 10 \mathrm{C}$ & 109.5 \\
\hline $\mathrm{O} 5-\mathrm{S} 1-\mathrm{O} 3$ & $112.19(7)$ & $\mathrm{H} 10 \mathrm{~B}-\mathrm{C} 10-\mathrm{H} 10 \mathrm{C}$ & 109.5 \\
\hline $\mathrm{O} 4-\mathrm{S} 1-\mathrm{O} 3$ & $111.96(7)$ & $\mathrm{C} 8-\mathrm{N} 1-\mathrm{C} 9$ & $114.35(16)$ \\
\hline $\mathrm{O} 5-\mathrm{S} 1-\mathrm{C} 1$ & $106.14(7)$ & $\mathrm{C} 8-\mathrm{N} 1-\mathrm{H} 3$ & $112.5(18)$ \\
\hline $\mathrm{O} 4-\mathrm{S} 1-\mathrm{C} 1$ & $105.83(7)$ & $\mathrm{C} 9-\mathrm{N} 1-\mathrm{H} 3$ & $109.5(19)$ \\
\hline $\mathrm{O} 3-\mathrm{S} 1-\mathrm{C} 1$ & $108.08(7)$ & $\mathrm{C} 8-\mathrm{N} 1-\mathrm{H} 4$ & $100.4(15)$ \\
\hline $\mathrm{C} 8-\mathrm{C} 7-\mathrm{H} 7 \mathrm{~A}$ & 109.5 & $\mathrm{C} 9-\mathrm{N} 1-\mathrm{H} 4$ & $115.9(15)$ \\
\hline $\mathrm{C} 8-\mathrm{C} 7-\mathrm{H} 7 \mathrm{~B}$ & 109.5 & $\mathrm{H} 3-\mathrm{N} 1-\mathrm{H} 4$ & $104(2)$ \\
\hline $\mathrm{C} 6-\mathrm{C} 1-\mathrm{C} 2-\mathrm{O} 1$ & $-178.78(14)$ & $\mathrm{C} 2-\mathrm{C} 1-\mathrm{C} 6-\mathrm{C} 5$ & $-1.0(2)$ \\
\hline
\end{tabular}




\section{supplementary materials}

$$
\begin{aligned}
& \mathrm{S} 1-\mathrm{C} 1-\mathrm{C} 2-\mathrm{O} 1 \\
& \mathrm{C} 6-\mathrm{C} 1-\mathrm{C} 2-\mathrm{C} 3 \\
& \mathrm{~S} 1-\mathrm{C} 1-\mathrm{C} 2-\mathrm{C} 3 \\
& \mathrm{O} 1-\mathrm{C} 2-\mathrm{C} 3-\mathrm{C} 4 \\
& \mathrm{C} 1-\mathrm{C} 2-\mathrm{C} 3-\mathrm{C} 4 \\
& \mathrm{C} 2-\mathrm{C} 3-\mathrm{C} 4-\mathrm{C} 5 \\
& \mathrm{C} 3-\mathrm{C} 4-\mathrm{C} 5-\mathrm{O} 2 \\
& \mathrm{C} 3-\mathrm{C} 4-\mathrm{C} 5-\mathrm{C} 6 \\
& \mathrm{O} 2-\mathrm{C} 5-\mathrm{C} 6-\mathrm{C} 1 \\
& \mathrm{C} 4-\mathrm{C} 5-\mathrm{C} 6-\mathrm{C} 1
\end{aligned}
$$

Hydrogen-bond geometry $\left(A,{ }^{\circ}\right)$
$4.4(2)$
$1.1(2)$
$-175.75(13)$
$179.34(17)$
$-0.6(3)$
$-0.2(3)$
$179.88(16)$
$0.4(3)$
$-179.29(14)$
$0.2(2)$

$$
\begin{aligned}
& 176.03(12) \\
& 0.19(13) \\
& 177.14(12) \\
& -119.17(12) \\
& 57.78(13) \\
& 120.73(11) \\
& -62.32(13) \\
& 179.41(18) \\
& -162.32(19)
\end{aligned}
$$

$D-\mathrm{H} \cdots A$

$163(2)$

$174(3)$

$138(2)$

$129(2)$

$171(2)$

$\mathrm{N} 1-\mathrm{H} 4 \cdots \mathrm{O} 5^{\text {iv }}$

$0.82(3)$

2.49 (3)

$3.084(2)$

$3.0691(19)$

$2.888(2)$

1.94 (3)

) $-y, x-y-1, z-1 / 3$. 
Fig. 1

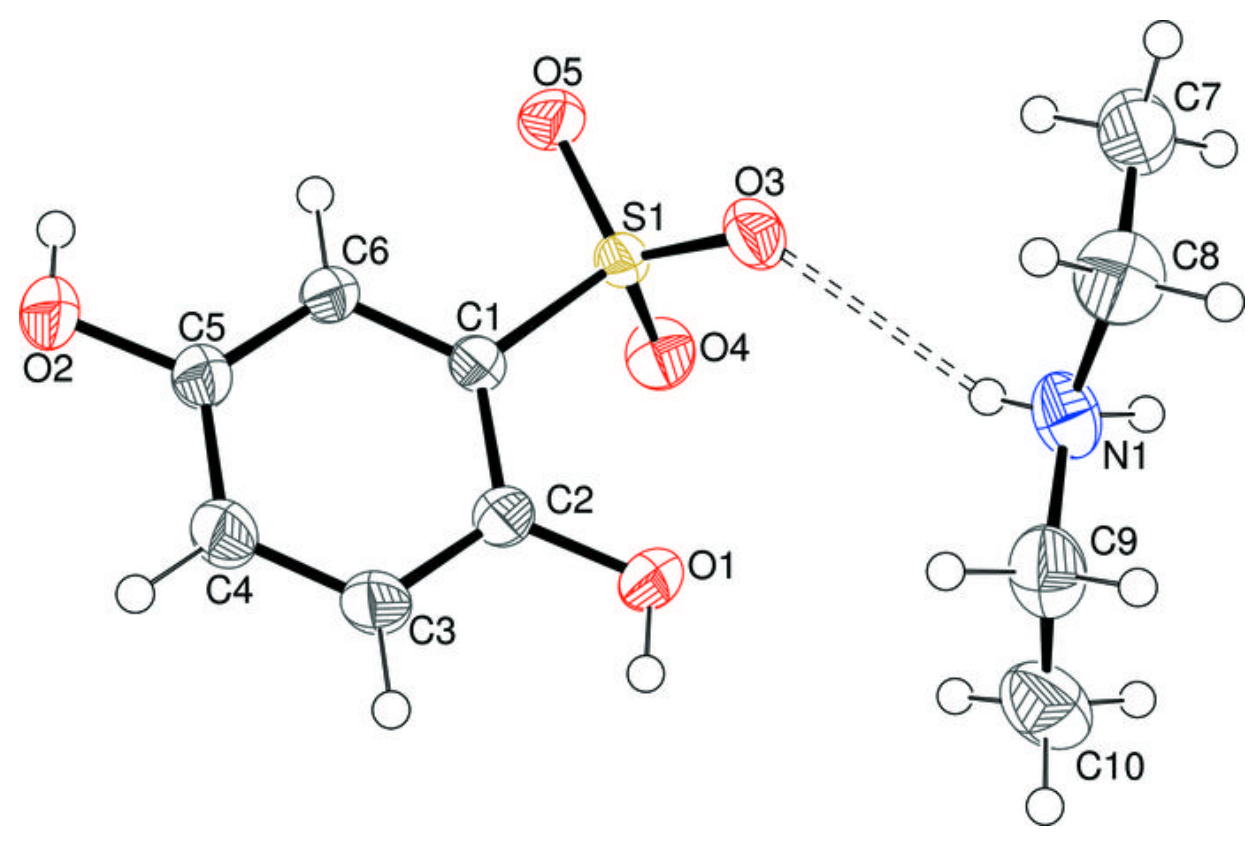


Fig. 2

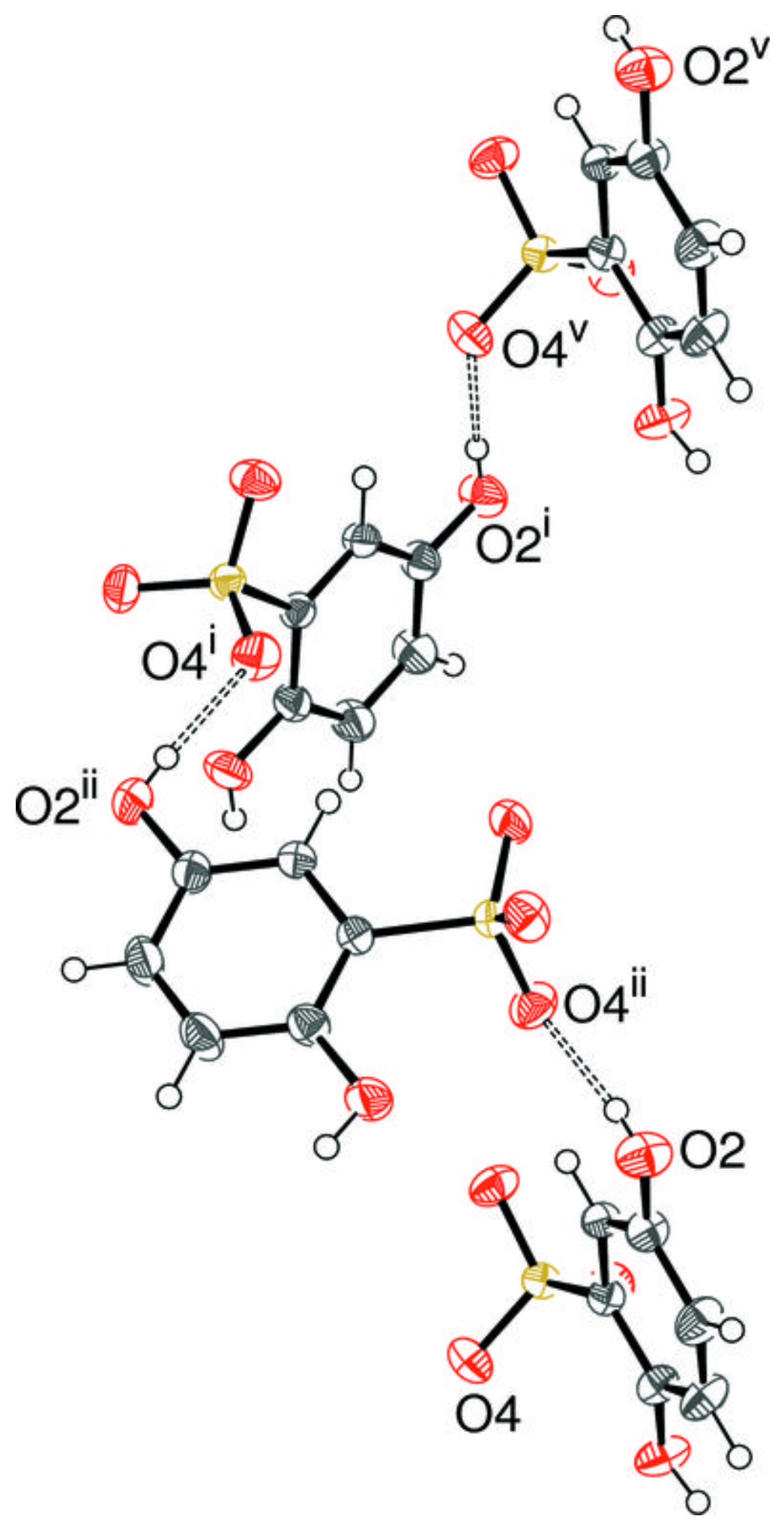

